\title{
Trial update: lo studio Diabetes REduction Approaches with ramipril and rosiglitazone Medications (DREAM)
}

\section{Trial update: the Diabetes REduction Approaches with ramipril and rosiglitazone Medications (DREAM) trial}

\author{
G. Gulli *
}

UOC Medicina Interna, AO Bianchi Melacrino Morelli, Reggio Calabria

\author{
KEY WORDS \\ Primary prevention \\ Lyfestile changes \\ ACE-inhibitors \\ Glitazones \\ 2-by-2 factorial design
}

\begin{abstract}
Summary BACKGround Diabetes (T2DM) is a worldwide medical and social emergency in the westernized societies. Lifestyle changes, diet and physical activity, have shown a protective effect on the likelihood of developing T2DM and are strongly recommended for primary prevention in people at increased risk for T2DM. However, maintaining adherence to these nonpharmacologic strategies is dishearteningly challenging, and the possible use of drugs to prevent T2DM remains of keen interest. Metformin, glitazones, acarbose and orlistat can reduce the risk of developing T2DM. Recently, post hoc analyses of several clinical studies suggested that ACE-I as well might reduce the risk of T2DM. AIM OF THE STUDY Since none of these trials was designed with this primary end-point, the Diabetes REduction Assessment with ramipril and rosiglitazone Medication (DREAM) Investigators studied the effects of ramipril or rosiglitazone on the risk of T2DM in a randomized trial designed with T2DM as a primary outcome. METHOD Subjects with fasting impaired plasma glucose levels or impaired glucose tolerance, without cardiovascular disease, were randomly assigned, in a 2-by-2 factorial design, to receive either ramipril (up to $15 \mathrm{mg}$ daily) or placebo and either rosiglitazone or placebo. They were followed for a median of 3 years. REsults Rates of the primary end points, T2DM or death, were not significantly lower in the ramipril group than in the placebo group. In contrast to ramipril, the use of rosiglitazone resulted in a significant reduction in T2DM or death. Discussıon Disappointingly, 14 participants on rosiglitazone developed non-fatal heart failure, compared with 2 cases in the placebo group, and the group gained 3\% $(2.2 \mathrm{~kg})$ more in body weight than the placebo group. Moreover, we still do not know whether the effect of rosiglitazone on the incidence of T2DM will persist after drug washout. ConcLusions We believe that the high cost of the drug and the study's negative cardiovascular and body weight outcomes favour a lifestyle strategy in preventing T2DM.
\end{abstract}

\section{Razionale}

Circa 194 milioni di persone, in tutto il mondo, sono affette da diabete [1]. Questa prevalenza globale fa del diabete una delle malattie non trasmissibili più frequenti. Nel 2002 il diabete è stato la quinta causa di morte negli Stati Uniti e ha determinato una spesa di 132 miliardi di dollari [2]. II diabete sta assumendo caratteristiche di epidemia in Paesi in via di sviluppo e in nazioni da poco industrializzate, e le proiezioni depongono per il raddoppio del numero mondiale di casi entro il 2030 [1]. Le sequele si-

\footnotetext{
* Corrispondenza:

Giovanni Gulli, UOC Medicina Interna,

AO Bianchi Melacrino Morelli, via Vallone Petrara,

89124 Reggio Calabria, e-mail: giovanni.gulli@virgilio.it
}

stemiche macroangiopatiche e microangiopatiche del diabete - ovvero la coronaropatia, la vasculopatia cerebrale e periferica, la neuropatia, la nefropatia, la retinopatia e le loro complicanze, infarto miocardico e scompenso cardia$\mathrm{co}$, ictus, disabilità, amputazioni, insufficienza renale terminale e cecità - riducono l'aspettativa di vita e aumentano la spesa sanitaria e assistenziale in ogni nazione.

Motivi medici ed economici rendono di straordinaria importanza lo sviluppo di efficaci strategie preventive del diabete mellito. Recenti studi hanno dimostrato come incisivi interventi sullo stile di vita possano ridurre il diabete incidente fino al $58 \%$, soprattutto in individui affetti da ridotta tolleranza al glucosio $[3,4]$, migliorando in modo duraturo il profilo di rischio cardiovascolare. Tuttavia, l'aderenza a lungo termine a uno stile di vita "prescritto" [5] e la sua fattibilità al di fuori di un protocollo di studio sono assai limita- 
te, e ciò ne preclude il ricorso estensivo. L'uso di farmaci, per quanto ambivalente (l'individuo, sentendosi "protetto" dal farmaco, tende a perseverare negli stili di vita inappropriati alla patologia che si vuole prevenire), potrebbe costituire un'alternativa efficace nei casi in cui gli interventi sullo stile di vita falliscano o non siano perseguibili.

Negli ultimi anni, studi condotti su una serie di farmaci (metformina, acarbose, troglitazone, pioglitazone, orlistat, rosiglitazone) [4,6-11] hanno dimostrato che la progressione al diabete può essere ridotta in soggetti con scarsa tolleranza ai carboidrati, e che tale riduzione si può osservare anche dopo la sospensione del farmaco, come nel braccio dello studio Diabetes Prevention Program (DPP) trattato con troglitazone. Una diminuzione dell'incidenza del diabete mediante l'uso di inibitori dell'enzima di conversione dell'angiotensina (ACE-I) è stata serendipicamente riscontrata in tre studi (Heart Outcomes Prevention Evaluation, HOPE; Prevention of Events with Angiotensin Converting Enzyme inhibition, PEACE; EURopean trial On reduction of cardiac events with Perindopril in stable coronary Artery disease investigators, EUROPA) [12-14] e confermata da una recente metanalisi di 12 studi randomizzati e controllati [15]. Tuttavia, in questi studi, l'ipotesi nulla non prevedeva la mancanza di differenza di diabete incidente fra i gruppi sottoposti a trattamento attivo 0 a placebo; si trattava, pertanto, di risultati derivati da analisi post hoc. Inoltre, in tali studi non erano eseguiti test di tolleranza orale al glucosio né all'inizio né alla fine dell'osservazione e la reale prevalenza di diabete era ignota in entrambe le fasi, inficiando la possibilità di stabilire la progressione a diabete da alterata tolleranza ai carboidrati. Da queste premesse epidemiologiche, scientifiche e statistiche ha tratto origine lo studio Diabetes REduction Approaches with ramipril and rosiglitazone Medications (DREAM).

\section{Metodi}

In questo studio, i cui risultati sono stati pubblicati nel New England Journal of Medicine (braccio ramipril) [16] e in Lancet (braccio rosiglitazone) [17], i ricercatori si sono posti due quesiti indipendenti: il primo, se il ramipril, alla dose di $15 \mathrm{~m} /$ die, prevenisse il diabete; il secondo, se il rosiglitazone, alla dose di $8 \mathrm{mg} / \mathrm{die}$, prevenisse il diabete. Gli autori hanno esaminato 24.952 volontari e selezionato 5.269 soggetti che soddisfacevano i criteri di inclusione: età > 30 anni, alterata glicemia a digiuno (IFPG, 110-125 $\mathrm{mg} / \mathrm{dL}$ ) o alterata tolleranza orale al glucosio (IGT, glicemia due ore dopo carico orale di $75 \mathrm{~g}$ di glucosio $\geq 140 \mathrm{ma}<$ $200 \mathrm{mg} / \mathrm{dL}$ ), assenza di storia personale di diabete (con l'eccezione del diabete gestazionale), malattia cardiovascolare, intolleranza agli ACE-inibitori o ai tiazolidinedioni. I partecipanti allo studio sono stati randomizzati, occultando l'assegnazione al gruppo di studio ai responsabili della selezione (la allocation concealed degli autori anglosassoni), secondo un disegno fattoriale $2 \times 2$ (Tab. 1) a ricevere ramipril o placebo e rosiglitazone o placebo a dosi progressivamente crescenti fino a quelle sopra riportate. Alla fine della randomizzazione, un quarto dei partecipanti allo studio ha assunto rosiglitazone, un quarto ramipril, un quarto sia rosiglitazone sia ramipril e un quarto solamente placebo. Gli esiti primari (primary outcomes) sono stati la diagnosi di diabete o il decesso, quest'ultimo inserito come esito primario per rendere ragione di un'eventuale maggiore incidenza di mancata diagnosi di diabete in chi muore rispetto a chi sopravvive. II periodo osservazionale mediano è stato di 3 anni.

\section{Risultati}

L'incidenza dell'outcome primario composito (insorgenza di diabete o decesso) non è risultata significativamente diversa $(p=0,15)$ tra il gruppo randomizzato a ramipril $(18,1 \%)$ e il gruppo randomizzato a placebo (19,5\%). I soggetti che hanno ricevuto ramipril, tuttavia, hanno manifestato una maggiore probabilità di andare incontro a regressione a normoglicemia rispetto a coloro che sono trattati con placebo (hazard ratio, $\mathrm{HR}=1,16$; intervallo di confidenza, $\mathrm{Cl}=1,07-1,27 ; \mathrm{p}=0,001$ ). Alla fine dello studio, il livello mediano di glicemia a digiuno non è risultato significativamente inferiore nel gruppo ramipril rispetto al gruppo placebo $(102,7 \mathrm{mg} / \mathrm{dL}$ vs $103,4 \mathrm{mg} / \mathrm{dL}, \mathrm{p}=$ 0,07 ), sebbene i livelli di glicemia, due ore dopo carico orale di $75 \mathrm{~g}$ di glucosio, fossero significativamente più bassi nel gruppo ramipril $(135,1 \mathrm{mg} / \mathrm{dL}$ vs $140,5 \mathrm{mg} / \mathrm{dL}, \mathrm{p}$ $=0,01$ ). Gli autori dello studio concludono che «ramipril può avere effetti favorevoli sul metabolismo del glucosio», ma che «per ora, l'uso routinario di ramipril con l'esplicito obiettivo di prevenire il diabete non è indicato».

Nel gruppo randomizzato a rosiglitazone, solamente 306 individui $(11,6 \%)$ hanno sviluppato l'outcome primario composito, rispetto a 686 individui $(26,0 \%)$ nel gruppo randomizzato a placebo $(\mathrm{HR}=0,40 ; \mathrm{Cl}=0,35-0,46 ; \mathrm{p}<$ $0,0001)$. Questa differenza non era determinata dal numero di decessi ( $H R=0,91 ; \mathrm{Cl}=0,55-1,49 ; \mathrm{p}=0,7)$, bensì

Tabella 1 Criterio di randomizzazione dei partecipanti allo studio DREAM

\begin{tabular}{clll}
\hline & & \multicolumn{2}{c}{ Fattore 1} \\
\cline { 2 - 3 } Fattore 2 & Rosiglitazone & Ramipril & Placebo \\
\cline { 2 - 3 } & Placebo & Ramipril e placebo & Placebo e rosiglitazone \\
\cline { 2 - 4 }
\end{tabular}


da un'ampia differenza nella frequenza del diabete ( $\mathrm{HR}=$ $0,38 ; \mathrm{Cl}=0,33-0,44 ; \mathrm{p}<0,0001)$ fra i due gruppi. Nel gruppo rosiglitazone, 1.330 soggetti $(50,5 \%)$ sono diventati normoglicemici, rispetto a soli $798(30,3 \%)$ nel gruppo placebo ( $\mathrm{HR}=1,71 ; \mathrm{Cl}=1,57-1,87 ; \mathrm{p}<0,0001)$. La frequenza degli eventi cardiovascolari (infarto miocardico, ictus, angina di nuova insorgenza, morte cardio-cerebrovascolare, procedure di rivascolarizzazione) è risultata sovrapponibile nei due gruppi; tuttavia 14 soggetti $(0,5 \%)$ nel gruppo rosiglitazone e $2(0,1 \%)$ nel gruppo placebo hanno sviluppato scompenso cardiaco $(p=0,01)$. Commentando questi risultati, gli autori affermano che «ogni 1.000 persone trattate con rosiglitazone per 3 anni, circa 144 casi di diabete saranno prevenuti, con un eccesso di 4-5 casi di scompenso cardiaco congestizio», ma che "occorrono ulteriori lavori per stabilire se i benefici effetti metabolici osservati con rosiglitazone porteranno a una riduzione delle complicanze cardiovascolari, renali, retiniche e di altre serie conseguenze per la salute».

\section{Discussione}

Lo studio DREAM, nella sua complessità, richiede valutazioni relative sia all'appropriatezza scientifica sia alle conseguenze pratiche. Se è vero che il ruolo degli ACE-I nella riduzione dell'incidenza di diabete di tipo 2 [12-15], seppur suggestivo, non era stato formalmente indagato bensì derivato da analisi post hoc, considerazione che conferisce piena valenza euristica al braccio ramipril dello studio, è altrettanto vero che numerosi studi avevano già documentato l'efficacia dei tiazolidinedioni (troglitazone, pioglitazone, rosiglitazone) nel ridurre la progressione a diabete in individui con scarsa tolleranza ai carboidrati ed elevato rischio di sviluppare diabete. Ciò priva il braccio rosiglitazone dello studio di parte del suo appeal. Tuttavia, sia le dimensioni sia le caratteristiche del campione, eterogeneo in termini di distribuzione geografica (anglosassoni, ispanici, neri, europidi, orientali) e bilanciato per età, genere, storia medica, uso di farmaci e caratteristiche fisiche, conferiscono agli esiti di questo studio un'importante valenza di definitività.

L'esito del ramipril nel ridurre il diabete incidente è stato inequivocabilmente negativo. Per le evidenze fisiopatologiche attuali, tale risultato non sorprende. II diabete di tipo 2 si manifesta clinicamente quando la cellula B-pancreatica non è più in grado di contrastare la ridotta sensibilità dei tessuti all'ormone, aumentando l'increzione insulinica. La presenza di recettori AT-1 per l'angiotensina II (AT II) è stata descritta sulla membrana delle cellule Bpancreatiche umane [18]. L'inibizione del sistema reninaangiotensina determinato dal ramipril non sembra indurre effetti clinicamente rilevanti sull'increzione insulinica, sebbene alcuni studi [19] abbiano documentato la riduzione dell'increzione insulinica, sia basale sia glucosio-stimolata, in corso di infusione di AT II vs placebo, verosimilmente mediata dalla soppressione della perfusione ematica del-
I'insula. Recettori AT-1 sono infatti presenti sia nel microcircolo delle insule sia nell'endotelio dei capillari del tessuto muscolare, maggiore responsabile della clearance insulino-stimolata del glucosio plasmatico. È quindi verosimile che uno stesso meccanismo, ovvero il miglioramento della perfusione ematica pancreatica e muscolare, renda conto del migliorato metabolismo del glucosio (esito secondario) osservato nel braccio ramipril pur a fronte di un mancato effetto "protettivo" sulla cellula B-pancreatica e, quindi, sulla progressione della malattia. Appare, quindi, eccessivamente difensiva la discussione sulle differenze fra i risultati di questo studio e gli studi precedenti che hanno suggerito, con analisi post hoc, una ridotta incidenza del diabete di tipo 2 dopo l'assunzione di ACE-I.

Per contro, due aspetti avrebbero meritato maggiore attenzione: la significativa riduzione dei valori pressori nei soggetti che assumevano $15 \mathrm{mg}$ di ramipril vs il gruppo di controllo (128/78 mmHg vs 132/80 mmHg, p < 0,001), non commentata, e la mancata diminuzione degli eventi cardiovascolari nel gruppo trattato, licenziata con la motivazione di una bassa frequenza di eventi nel corso dello studio, tale da non consentire il conseguimento di un'eventuale significatività statistica. Entrambi i risultati contrastano con i dati dello studio HOPE, nel quale la mancanza di significatività della differenza dei valori pressori osservata a fine studio nei due gruppi (ramipril e placebo) amplificava il dato della significativa riduzione di eventi cardiovascolari nel gruppo trattato con ramipril. Questo dato induceva gli autori, e fra questi lo stesso Salim Yusuf, coprincipal investigator di entrambe le ricerche, HOPE e DREAM, ad affermare che «è verosimile che gli inibitori dell'enzima di conversione dell'angiotensina esercitino meccanismi diretti aggiuntivi sul cuore o sui vasi, che sono importanti» ma che, in questo studio, non si sono apprezzati, pur in una popolazione a rischio cardiovascolare aumentato [20].

I risultati del braccio rosiglitazone sono di più difficile interpretazione e richiedono una pregiudiziale valutazione semantica. È noto, infatti, che i tiazolidinedioni possono migliorare il controllo glicemico nei pazienti affetti da diabete di tipo 2 sia migliorando la sensibilità all'insulina sia preservando dal declino funzionale la cellula B-pancreatica [21], ed è ragionevole presumere che possano esplicare la stessa azione nelle fasi precedenti la manifestazione clinica della malattia, di fatto ritardandola. Se "ritardare" è accettato come sinonimo di "prevenire" (la lingua inglese consente tale ambivalenza), allora il rosiglitazone ha indiscutibilmente ridotto la frequenza di diabete incidente. Se ciò significhi che, dopo una media di 3 anni di assunzione del farmaco, la malattia non si presenterà perché il rosiglitazone ha agito come disease modifying drug e che, quindi, l'eccesso di mortalità cardio-cerebrovascolare che contraddistingue la patologia diabetica conclamata sarà abolito, è un quesito al quale lo studio non può dare risposta. I dati che lo studio fornisce, di fatto, non supportano questa ipotesi: la frequenza degli eventi cardiovascolari (infarto miocardico, ictus, angina di nuova insorgenza, morte car- 
dio-cerebrovascolare, procedure di rivascolarizzazione) è risultata sovrapponibile nei due gruppi. Se il razionale della prevenzione del diabete consiste nella prevenzione della mortalità e morbilità cardiovascolare, la prevenzione offerta da 3 anni di terapia con rosiglitazone è stata nulla; questa considerazione rende assai opinabile l'opzione di trattare con un farmaco ipoglicemizzante individui con glicemie non diabetiche. Se si accetta il dato fornito da studi osservazionali [22] di una riduzione del $20 \%$ del rischio cardio-cerebrovascolare per ogni decremento di $20 \mathrm{mg} / \mathrm{dL}$ della glicemia a digiuno, i $9 \mathrm{mg} / \mathrm{dL}$ di differenza, a fine studio, a favore del gruppo trattato con rosiglitazone, dovrebbero indurre una riduzione del rischio di circa il $9 \%$. Come sottolineato in un editoriale di Lancet pubblicato online contestualmente ai risultati dello studio [23], per prevenire un evento si dovrebbero trattare per 3 anni 554 individui, con una spesa di 1.376.617 euro.

A complicare l'interpretazione dei risultati del braccio rosiglitazone dello studio DREAM concorrono due effetti dovuti alla farmacodinamica del composto, ovvero l'incremento di peso e l'aumentata incidenza di scompenso cardiaco, e il confronto con i risultati del Finnish Diabetes Prevention Study $[24,25]$.

L'incremento ponderale, pur essendo interpretato dagli autori come metabolicamente vantaggioso, poiché si verifica prevalentemente come accumulo di adipe nelle regioni femoro-gluteali, con conseguente riduzione del rapporto vita-fianchi, dall'analisi dei dati non sembra raggiungere un plateau. Non si possono prevedere gli effetti endocrini e metabolici a lungo termine di tale progressivo incremento ponderale, mentre sono dimostrati sia la riduzione dell'attività fisica che si accompagna all'aumento di peso [26] sia l'aumento del rischio cardio-cerebrovascolare che la sedentarietà comporta. Incidentalmente, in un altro studio pubblicato successivamente [27], il rosiglitazone non ha confermato una più favorevole distribuzione dell'adipe progressivamente accumulato. Questo studio, inoltre, ha ribadito l'aumentata incidenza di scompenso cardiaco indotta dal rosiglitazone e individuato un incremento di fratture nel gruppo trattato. Sul piano squisitamente prasseologico è, pertanto, legittimo porsi la domanda sull'opportunità di somministrare a una persona con ridotta tolleranza al glucosio o con alterata glicemia a digiuno un farmaco che determina aumento di peso e che espone al rischio di scompenso cardiaco e forse di frattura per prevenire (o ritardare) l'esordio di una malattia, quando è stato ampiamente documentato che le modificazioni dello stile di vita (riduzione dell'apporto calorico, incremento dell'attività fisica) possono sortire lo stesso effetto, con un number needed to treat (NNT) paragonabile, in modo estremamente più economico e sostenuto nel tempo.

Relativamente a quest'ultimo punto, il silenzio che ha seguito la presentazione dei dati dello studio di washout (valutazione del perdurare dell'effetto del rosiglitazone dopo la sospensione del farmaco) durante il Congresso della International Diabetes Federation (IDF), tenutosi in novembre a Cape Town, lascia supporre che, come già osservato per il braccio troglitazone del DPP [7], la sospensione del trattamento con rosiglitazone abbia annullato la riduzione della frequenza di progressione a diabete.

Infine, è dovuto un breve commento di ordine farmacoeconomico sull'affermazione conclusiva dello studio: «Ogni 1.000 persone trattate con rosiglitazone per 3 anni, circa 144 casi di diabete saranno prevenuti». In Italia il prezzo di una compressa di rosiglitazone da $8 \mathrm{mg}$ è di 2,269 euro; il costo per la prevenzione farmacologica di 144 casi di diabete ammonterebbe a 2.484.555 euro. A fronte della documentata efficacia degli interventi sullo stile di vita, in assenza di indicatori certi di progressione di malattia, in termini di costo/efficacia un intervento di tale portata non sembra essere proponibile.

\section{Conclusioni}

Lo studio DREAM ha richiesto un formidabile sforzo organizzativo per esaminare circa 25.000 individui in 191 centri di ricerca clinica in 21 differenti nazioni, reclutarne oltre 5.000 e seguirli per più di 3 anni. II completamento dello studio, ben disegnato, è per sé un successo rispettabile. Nel valutare uno studio di tale portata è importante ricordare che i trial clinici sono condotti per rispondere, con metodo scientifico, a un quesito estremamente specifico. Lo studio DREAM ha dimostrato che il ramipril non previene/ritarda la progressione a diabete, mentre il rosiglitazone ha un'indiscutibile efficacia nel prevenire/ritardare l'insorgenza della malattia. La trasposizione di questa conoscenza dalla ricerca clinica alla pratica clinica non è né automatica né immediata. Solamente la revisione complessiva dei dati, integrati con quelli generati dalla misurazione degli outcome principali e degli effetti collaterali a due anni dalla sospensione del farmaco, consentirà di utilizzare correttamente questa informazione.

\section{Bibliografia}

[1] Wild S, Roglic G, Green A, Sicree R, King H. Global prevalence of diabetes: estimates for the year 2000 and projections for 2030. Diabetes Care 2004;27(5):1047-53.

[2] Hogan P, Dall T, Nikolov P; American Diabetes Association. Economic costs of diabetes in the US in 2002. Diabetes Care 2003;26(3):917-32.

[3] Tuomilehto J, Lindstrom J, Eriksson JG, et al. Prevention of type 2 diabetes mellitus by changes in lifestyle among subjects with impaired glucose tolerance. N Engl J Med 2001; 344(18):1343-50.

[4] Knowler WC, Barrett-Connor E, Fowler SE, et al; Diabetes Prevention Program Research Group. Reduction in the incidence of type 2 diabetes with lifestyle intervention or metformin. N Engl J Med 2002;346(6):393-403.

[5] Methods for voluntary weight loss and control. NIH Technology Assessment Conference Panel. Consensus Development Conference, 30 March to 1 April 1992. Ann Intern Med 1993;119(7 Pt 2):764-70.

[6] Chiasson JL, Josse RG, Gomis R, Hanefeld M, Karasik A, 
Laakso M; STOP-NIDDM Trail Research Group. Acarbose for prevention of type 2 diabetes mellitus: the STOP-NIDDM randomised trial. Lancet 2002;359(9323):2072-7.

[7] Knowler WC, Hamman RF, Edelstein SL, et al; Diabetes Prevention Program Research Group. Prevention of type 2 diabetes with troglitazone in the Diabetes Prevention Program. Diabetes 2005;54(4):1150-6.

[8] Buchanan TA, Xiang AH, Peters RK, et al. Preservation of pancreatic beta-cell function and prevention of type 2 diabetes by pharmacological treatment of insulin resistance in high-risk hispanic women. Diabetes 2002;51(9):2796-803.

[9] Durbin RJ. Thiazolidinedione therapy in the prevention/delay of type 2 diabetes in patients with impaired glucose tolerance and insulin resistance. Diabetes Obes Metab 2004; 6(4):280-5.

[10] Heymsfield SB, Segal KR, Hauptman J, et al. Effects of weight loss with orlistat on glucose tolerance and progression to type 2 diabetes in obese adults. Arch Intern Med 2000; 160(9):1321-6.

[11] Xiang AH, Peters RK, Kjos SL, et al. Effect of pioglitazone on pancreatic beta-cell function and diabetes risk in Hispanic women with prior gestational diabetes. Diabetes 2006; 55(2):517-22.

[12] Yusuf S, Sleight P, Pogue J, Bosch J, Davies R, Dagenais G. Effects of an angiotensin-converting-enzyme inhibitor, ramipril, on cardiovascular events in high-risk patients. The Heart Outcomes Prevention Evaluation Study Investigators. N Engl J Med 2000;342(3):145-53.

[13] Braunwald E, Domanski MJ, Fowler SE, et al; PEACE Trial Investigators. Angiotensin-converting-enzyme inhibition in stable coronary artery disease. N Engl J Med 2004;351(20): 2058-68.

[14] Fox KM; EURopean trial On reduction of cardiac events with Perindopril in stable coronary Artery disease Investigators. Efficacy of perindopril in reduction of cardiovascular events among patients with stable coronary artery disease: randomised, double-blind, placebo-controlled, multicentre trial (the EUROPA study). Lancet 2003;362(9386):782-8.

[15] Abuissa H, Jones PG, Marso SP, O'Keefe JH Jr. Angiotensinconverting enzyme inhibitors or angiotensin receptor blockers for prevention of type 2 diabetes: a meta-analysis of randomized clinical trials. J Am Coll Cardiol 2005;46(5):821-6.

[16] DREAM Trial Investigators; Bosch J, Yusuf S, Gerstein HC, et al. Effect of ramipril on the incidence of diabetes. N Engl J Med 2006;355(15):1551-62.
[17] DREAM Trial Investigators; Gerstein HC, Yusuf S, Bosch J, et al. Effect of rosiglitazone on the frequency of diabetes in patients with impaired glucose tolerance or impaired fasting glucose: a randomised controlled trial. Lancet 2006;368 (9541):1096-105.

[18] Tahmasebi M, Puddefoot JR, Inwang ER, Vinson GP. The tissue renin-angiotensin system in human pancreas. J Endocrinol 1999;161(2):317-22.

[19] Fliser D, Schaefer F, Schmid D, Veldhuis JD, Ritz E. Angiotensin II affects basal, pulsatile, and glucose-stimulated insulin secretion in humans. Hypertension 1997;30(5): 1156-61.

[20] Haffner SM, Stern MP, Hazuda HP, Mitchell BD, Patterson JK. Cardiovascular risk factors in confirmed prediabetic individuals. Does the clock for coronary heart disease start ticking before the onset of clinical diabetes? JAMA 1990; 263(21):2893-8.

[21] Wallace TM, Levy JC, Matthews DR. An increase in insulin sensitivity and basal beta-cell function in diabetic subjects treated with pioglitazone in a placebo-controlled randomized study. Diabet Med 2004;21(6):568-76.

[22] Levitan EB, Song Y, Ford ES, Liu S. Is nondiabetic hyperglycemia a risk factor for cardiovascular disease? A metaanalysis of prospective studies. Arch Intern Med 2004;164 (19):2147-55.

[23] Tuomilehto J, Wareham N. Glucose lowering and diabetes prevention: are they the same? Lancet 2006;368(9543): 1218-9.

[24] Laaksonen DE, Lindstrom J, Lakka TA, et al; Finnish Diabetes Prevention Study Group. Physical activity in the prevention of type 2 diabetes: the Finnish Diabetes Prevention Study. Diabetes 2005;54(1):158-65.

[25] Lindstrom J, Ilanne-Parikka P, Peltonen M, et al; Finnish Diabetes Prevention Study Group. Sustained reduction in the incidence of type 2 diabetes by lifestyle intervention: followup of the Finnish Diabetes Prevention Study. Lancet 2006; 368(9548):1673-9.

[26] Petersen L, Schnohr P, Sorensen TI. Longitudinal study of the long-term relation between physical activity and obesity in adults. Int J Obes Relat Metab Disord 2004;28(1): 105-12.

[27] Kahn SE, Haffner SM, Heise MA, et al; ADOPT Study Group. Glycemic durability of rosiglitazone, metformin, or glyburide monotherapy. N Engl J Med 2006;355(23):2427-43. 